\title{
Effect of rifampicin in the treatment of pruritus in hepatic cholestasis
}

\author{
G V Gregorio, C S Ball, A P Mowat, G Mieli-Vergani
}

\begin{abstract}
Pruritus in hepatic cholestasis has been suggested to be secondary to a high concentration of serum bile acids. Rifampicin, which inhibits the uptake of bile acids by hepatocytes, has been used to treat pruritus. To determine the efficacy of rifampicin as a treatment for refractory pruritus, the medical records of 33 children (median age 25 months, range 4-135; 19 boys) with chronic cholestatic liver disease (21 with Alagille's syndrome, eight with progressive intrahepatic cholestasis, one with extrahepatic biliary atresia, one with an inborn error of bile acid metabolism, and one with cryptogenic cirrhosis) were reviewed restrospectively. The median dose of rifampicin was $5(4-10) \mathrm{mg} / \mathrm{kg} / \mathrm{day}$. The median duration of intake was 36 (4-120) weeks.

Complete relief of pruritus was noted in five (15\%) patients and a partial response in 12 $(36 \%)$. Overall, no significant difference was noted in the laboratory parameters before and after treatment with rifampicin. In the 21 patients with Alagille's syndrome, however, a significant decrease in alkaline phosphatase was seen before and after one and six months of starting treatment. No adverse side effects were seen.

Rifampicin appears to be effective in the treatment of refractory pruritus. A prospective study is warranted to assess further the effect of rifampicin treatment in children with hepatic cholestasis.

(Arch Dis Child 1993; 69: 141-143)
\end{abstract}

Pruritus in hepatic cholestasis is a major disabling symptom which interferes with the daily activities and sleep of patients. ${ }^{1}$ The mechanism of pruritus is uncertain, though it is believed that the high concentration of bile acids causes hepatocyte membrane injury and triggers the release of pruritogenic substances. Several drugs have been used with variable success. The range of recommended treatment includes cholestyramine, ${ }^{2}$ phenobarbitone ${ }^{3}$ ursodeoxycholic acid, ${ }^{+}$ androgenic steroids, ${ }^{5}$ naloxone, ${ }^{6}$ histamine antagonists, ${ }^{7}$ plasmapheresis, ${ }^{8}$ and phototherapy. In pruritus which is unresponsive to medical management, the partial external diversion of bile has been proposed for patients with progressive intrahepatic cholestasis. ${ }^{9}$

Rifampicin, an antimicrobial drug widely used in the treatment of tuberculosis, has been shown to be effective against pruritus. Podesta et al have shown in a double blind placebo controlled study of 14 patients with primary biliary cirrhosis that the drug at $600 \mathrm{mg} /$ day gave complete relief of pruritus in $11(79 \%)$ and partial response in three $(21 \%)$ patients. ${ }^{10}$ In a similar double blind crossover study in children with chronic cholestatic liver disease, ${ }^{11}$ rifampicin at $10 \mathrm{mg} / \mathrm{kg} /$ day was likewise shown to be effective in alleviating pruritus in all five patients.

The effect of treatment with rifampicin has been attributed to its ability to enhance the activity of the mixed function oxidase system and increasing the content of the hepatic cytochrome $P-450 .{ }^{12}$ These promote $6-\alpha$ hydroxylation and subsequent 6- $\alpha$ glucoronidation of bile acids, thus decreasing the pool of toxic bile acids and facilitating the synthesis of protective acids. ${ }^{13}$ It has also been suggested that because of its antimicrobial action, rifampicin modifies the synthesis of secondary bile acids in the intestinal lumen and consequently reduces the amount of hepatotoxic lithocolic bile acids.

At the paediatric liver service, King's College Hospital, rifampicin has been used to treat pruritus in children with hepatic cholestasis which was refractory to cholestyramine, phenobarbitone, ursodeoxycholic acid, antihistamines, or ultraviolet treatment. The objective of this study was to determine whether rifampicin was an effective treatment in these patients.

\section{Patients and methods}

A retrospective analysis was made of the medical records of all 33 patients who were given rifampicin as a treatment for pruritus. The median age of the patients was $25(4-135)$ months at the time the drug was given (table 1). Twenty one had Alagille's syndrome (64\%) and eight had progressive intrahepatic cholestasis (25\%). All patients had intractable pruritus (continuous pruritus disturbing sleep) not relieved by other drugs. The median duration of symptoms was 14 (1-112) months before rifampicin treatment.

Table 1 Clinical features of 33 patients before treatment with rifampicin. Values are No(\%) unless stated otherwise

\begin{tabular}{lc}
\hline $\begin{array}{l}\text { Median (range) age when drug was given } \\
\text { (months) }\end{array}$ & $24 \cdot 9(4 \cdot 2-135 \cdot 0)$ \\
Sex & \\
Male & $19(58)$ \\
Female & $14(42)$ \\
Diagnosis of liver disease & $21(64)$ \\
Alagille's syndrome & $8(24)$ \\
Progressive intrahepatic cholestasis & $1(3)$ \\
Extrahepatic biliary atresia & $1(3)$ \\
Giant cell hepatitis & $1(3)$ \\
Inborn error of bile acid metabolism & $1(3)$ \\
Cryptogenic cirrhosis & $6 \cdot 0(2 \cdot 0-60 \cdot 0)$ \\
Median (range) age of onset of pruritus & $13 \cdot 8(1 \cdot 0-111 \cdot 6)$ \\
(months) & \\
Median (range) duration of pruritus before & \\
drug treatment (months) & $28(85)$ \\
Treatment before rifampicin & $12(36)$ \\
Cholestyramine & $10(30)$ \\
Antihistamines & $9(27)$ \\
Phenobarbitone & $1(3)$ \\
Ultraviolet & \\
Ursodeoxycholic acid & \\
\hline
\end{tabular}

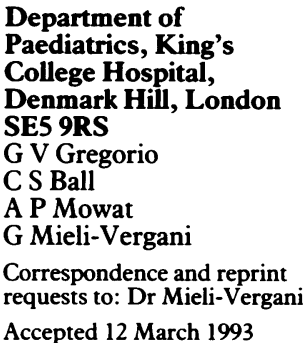


Table 2 Laboratory parameters of the 33 patients before rifampicin treatment. Values are median (range)

\begin{tabular}{|c|c|}
\hline Parameter & Value \\
\hline $\begin{array}{l}\text { Total bilirubin }(\mu \mathrm{mol} / \mathrm{l}) \\
\text { AST }(\mathrm{IU} / \mathrm{l})^{\star} \\
\text { GGT }(\mathrm{IU} / \mathrm{l})^{\star} \\
\text { Alkaline phosphatase }(\mathrm{IU} / \mathrm{l}) \\
\text { Albumin }(\mathrm{g} / \mathrm{l}) \\
\text { Cholesterol }(\mathrm{mmol} / \mathrm{l})(\mathrm{n}=30)\end{array}$ & $\begin{array}{l}115(4-275) \\
184(52-615) \\
306(13-1420) \\
763(320-2395) \\
41(30-54) \\
10 \cdot 9(2 \cdot 4-38 \cdot 5)\end{array}$ \\
\hline
\end{tabular}

Response to treatment was assessed as: (a) complete if pruritus stopped; (b) partial, if there was a decrease in intensity but persistence of the pruritus; or (c) negative, if there was no improvement in the symptom. Biochemical tests of liver function and serum cholesterol concentration before treatment and at one, three, and six months after starting treatment with rifampicin were analysed.

Descriptive analysis was performed and the data were expressed as median (range) values. Comparison of continuous data was carried out using the non-parametric Wilcoxon rank sum test. The $\chi^{2}$ test was used for discrete variables. A value of $p<0.05$ was considered significant.

\section{Results}

Rifampicin was given once or twice daily at a median dose of $5(4-10) \mathrm{mg} / \mathrm{kg} /$ day. The median duration of intake was 32 (4-102) weeks.

Median levels of bilirubin, liver enzymes, and cholesterol before rifampicin treatment were all increased (table 2). Increased levels of bilirubin $(>20 \mu \mathrm{mol} / \mathrm{l})$ were seen in $28(85 \%)$ patients. All patients had an alkaline phosphatase of $>300$ IU/l. Marked hypercholesterolaemia $(>15 \mathrm{mmol} / \mathrm{l})$ was shown in 10 of $30(30 \%)$ patients, all of whom had Alagille's syndrome.

In the total series of patients no significant differences were noted in the laboratory parameters before and after one, three, and six months of rifampicin treatment. Among the 21 patients with Alagille's syndrome, however, a significant decrease in alkaline phosphatase was shown before and after one and six months of starting treatment with rifampicin (table 3 ).

Complete relief of pruritus was noted in five (15\%) patients whereas a partial response was shown in $12(36 \%)$. No beneficial effect was seen in $16(48 \%)$ patients.

Table 3 Laboratory indices before and one, three, and six months after rifampicin treatment in the 21 patients with Alagille's syndrome. Data expressed as median (range)

\begin{tabular}{lllll}
\hline & $\begin{array}{l}\text { Baseline } \\
(n=21)\end{array}$ & $\begin{array}{l}\text { After one month } \\
(n=21)\end{array}$ & $\begin{array}{l}\text { After three months } \\
(n=18)\end{array}$ & $\begin{array}{l}\text { After six months } \\
(n=17)\end{array}$ \\
\hline Total bilirubin $(\mu \mathrm{mol} / \mathrm{l})$ & $126(15-257)$ & $138(28-327)$ & $132(10-308)$ & $109(20-310)$ \\
AST $(\mathrm{IU} /)^{\star}$ & $200(75-615)$ & $200(53-666)$ & $210(70-488)$ & $204(68-463)$ \\
GGT (IU/) & $418(42-1420)$ & $385(24-1294)$ & $440(27-885)$ & $453(21-872)$ \\
Alkaline phosphatase (IU/l) & $972(509-2395)$ & $773(368-2510) \dagger$ & $802(406-1910)$ & $809(499-1647) \ddagger$ \\
Albumin $(\mathrm{g} / \mathrm{l})$ & $41(30-49)$ & $42(30-46)$ & $42(29-50)$ & $42(34-48)$ \\
Cholesterol (mmol/l) & $12 \cdot 9(2 \cdot 4-38 \cdot 5)$ & $13(3-52)$ & $14 \cdot 9(2 \cdot 6-46 \cdot 9)$ & $13 \cdot 1(2 \cdot 9-28 \cdot 8)$ \\
\hline
\end{tabular}

$\star A S T=$ serum aspartate transaminase; GGT $=\gamma$-glutamyltransferase

Significant difference noted before and after one month of rifampicin treatment; $p<0.003$ ISignificant difference noted before and after six months of rifampicin treatment; $p<0.02$.
Table 4 Response to rifampicin according to the underlying liver disease

\begin{tabular}{llll}
\hline & \multicolumn{2}{l}{ Response } \\
\cline { 2 - 4 } Liver disease & Complete & Partial & Negative \\
\hline $\begin{array}{l}\text { Alagille's syndrome }(\mathrm{n}=21) \\
\begin{array}{l}\text { Progressive intrahepatic } \\
\text { cholestasis }(\mathrm{n}=8)\end{array}\end{array}$ & 3 & 7 & 11 \\
$\begin{array}{c}\text { Extrahepatic biliary atresia } \\
(\mathrm{n}=1)\end{array}$ & 0 & 3 & 5 \\
$\begin{array}{l}\text { Giant cell hepatitis }(\mathrm{n}=1) \\
\text { Inborn error of bile acid } \\
\text { metabolism }(\mathrm{n}=1)\end{array}$ & 0 & 1 & 0 \\
\begin{tabular}{l} 
Cryptogenic cirrhosis $(\mathrm{n}=1)$ \\
\hline
\end{tabular} & 1 & 1 & 0 \\
\hline
\end{tabular}

No significant differences in the response according to the underlying liver disease $(\mathrm{p}>0.05)$.

Table 5 Response to drugs used for the treatment of pruritus

\begin{tabular}{lllll}
\hline \multicolumn{5}{c}{ Response } \\
\cline { 2 - 4 } Drug & Complete & Partial & Negative & Total \\
\hline RMP alone & 2 & 4 & 3 & $9(27 \cdot 2)$ \\
RMP+UDCA & 0 & 3 & 0 & $3(9 \cdot 1)$ \\
RMP+cholestyramine & 2 & 3 & 5 & $10(30 \cdot 3)$ \\
RMP+phenobarbitone & 1 & 1 & 0 & $2(6 \cdot 1)$ \\
RMP+antihistamines & 0 & 0 & 1 & $1(3 \cdot 0)$ \\
RMP+two other drugs & 0 & 1 & 7 & $8(24 \cdot 2)$
\end{tabular}

$\mathrm{RMP}=$ rifampicin, $\mathrm{UDCA}=$ ursodeoxycholic acid.

tOther drugs include a combination of UDCA, phenobarbitone, or antihistamines.

No significant difference in the response whether the patient was receiving rifampicin alone or rifampicin with other drug $(\mathrm{p}>0.05)$.

Response to treatment was not related to the underlying liver disease (table 4), intake of concomitant drugs (table 5), or degree of abnormality of the liver function tests and cholesterol.

No patient developed adverse clinical features or changes in laboratory phenobarbitone indices on treatment with rifampicin.

\section{Discussion}

Pruritus in hepatic cholestasis remains a therapeutic challenge with a significant morbidity and a poorly defined pathogenesis. The degree of pruritus can range from mild and intermittent to severe and intractable, which causes serious disability and discomfort.' Pruritus is usually generalised but most severely affects the palms and soles, the extensor surface of the arms and legs, and the upper trunk. Although there is no direct evidence that retained bile acids on the nerve endings of the skin account for the pruritus, current modalities of treatment have been directed towards decreasing the serum concentration of bile acids.

One of the drugs known to inhibit the uptake of bile acids by the hepatocyte is rifampicin. ${ }^{13}$ The efficacy of rifampicin as a drug for the treatment of pruritus was first described by Ghent and Carruthers in a double blind, randomised crossover trial in nine patients with primary biliary cirrhosis who were all unresponsive to cholestyramine. ${ }^{14}$ Rifampicin was given at a dose of $300-450 \mathrm{mg} /$ day and the effect was evident after the first week of treatment. Bachs and Pares have likewise shown that rifampicin is more effective than phenobarbitone in alleviating pruritus associated with primary biliary cirrhosis. ${ }^{3}$ 
Our study in 33 children with a variety of cholestatic liver diseases has shown that rifampicin at a median dose of $5(4-10) \mathrm{mg} / \mathrm{kg} /$ day was successful in $17(52 \%)$ patients in producing a complete or partial relief of pruritus. All these patients had intractable pruritus not relieved by other drugs such as cholestyramine, phenobarbitone, ursodeoxycholic acid, antihistamines, or ultraviolet treatment. In an earlier report, Cynamon et al showed that rifampicin was effective in the treatment of pruritus in $100 \%$ of patients. " This study, however, used only five children, aged 1-17 years, diagnosed to have non-syndromic paucity of the intrahepatic bile ducts (two children), progressive intrahepatic cholestasis (two children), and extrahepatic biliary atresia (one child). In our series, the response was noted in 10 patients with Alagille's syndrome, three with progressive intrahepatic cholestasis, and one patient each with extrahepatic biliary atresia, giant cell hepatitis, inborn error of bile acid metabolism, and cryptogenic cirrhosis.

Although rifampicin has been shown to enhance the hepatic microsomal drug oxidising system leading to drug interactions when given simultaneously with phenobarbitone, ${ }^{12}$ ursodeoxycholic acid, cholestyramine, or antihistamines, there was no significant difference in the response whether the patient was receiving rifampicin alone or on other drugs for the treatment of pruritus.

In the total series, there was no improvement in the biochemical indices before and after treatment with rifampicin. In the 21 patients with Alagille's syndrome, however, a significant decrease in alkaline phosphate was noted after one and six months of treatment with rifampicin. This finding confirms a previous report by Bachs and Pares $^{3}$ who also showed an improvement in alkaline phosphatase, in addition to the serum bile acids and $\gamma$ glutamyl transpeptidase levels after rifampicin treatment of patients with primary biliary cirrhosis. The effect has been attributed to the ability of rifampicin to induce microsomal activity. Although the drug has been shown to compete with the uptake of organic anions by the liver and impair biliary excretion, causing an increase in both unconjugated and conjugated forms of bilirubin, ${ }^{15} 16$ we observed no significant difference in the total bilirubin concentrations before and after treatment with rifampicin.

No adverse clinical features or changes in biochemical parameters were noted in our patients. Treatment with rifampicin in a patient with primary biliary cirrhosis has been reported to cause severe haemolytic anaemia and renal failure. ${ }^{3}$ Adverse hepatic ${ }^{16}$ and immunoallergic effects ${ }^{17}$ have also been described. The immunosuppressive effects of rifampicin and the theoretical possibility of the emergence of resistant organisms should also be considered during prolonged administration of the drug, ${ }^{18}$ though none of these side effects were observed during the follow up of our patients.

In summary, this study has shown that rifampicin is effective in $52 \%$ of children with cholestatic liver disease in causing a complete or partial relief of pruritus. An improvement in alkaline phosphatase after one and six months of starting rifampicin treatment was shown in the patients with Alagille's syndrome. No adverse reactions were noted. A double blind, randomised, placebo controlled trial should be performed to further assess the efficacy and safety of rifampicin in the treatment of pruritus in children with hepatic cholestasis.

1 Turgeon DK, Watkins PB. Pruritus in chronic cholestasis: evidence against involvement of cytochrome P450IIIA. evidence against involvement
Gastroenterology 1990; 98: A641.

2 Carey J, Williams G. Relief of pruritus of jaundice with a bile acid sequestering resin. $\mathcal{F} A M A$ 1961; 176: 432-5.

3 Bachs L, Pares A, Elena M, Piera C, Rodes J. Comparison of rifampicin with phenobarbitone for treatment of pruritus in biliary cirrhosis. Lancet 1989; ii: 574-6.

4 Poupon RE, Balkau B, Eschwege E, Poupon R. A multicenter, controlled trial of ursodiol for the treatment of primary biliary cirrhosis. N Engl F Med 1991; 324: 1548-54.

5 Walt RP, Daneshmend TK, Fellows IW, Toghill PJ. Effect of stanozolol on itching in primary biliary cirrhosis. BMF 1988; 296: 607 .

6 Bergasa NV, Schmitt JS, Talbot T, et al. Naloxone ameliorates pruritus of cholestasis in a pilot controlled trial. Gastropruritus of cholestasis in
enterology 1990; 98: A569.

7 Garden J, Ostrow JD, Roenigk HH. Pruritus in hepatic cholestasis. Pathogenesis and therapy. Arch Dermatol 1985; 121: 1415-20.

8 Turnberg LA, Mahoney MP, Gleeson MH. Plasmapheresis and plasma exchange in the treatment of hyperlipaemia and xanthomatous neuropathy in patients with primary biliary cirrhosis. Gut 1972; 13: 976-81.

9 Cerio R, Murphy GM, Sladen GE, MacDonald DM. A combination of phototherapy and cholestyramine for the relief of pruritus in patients with primary biliary cirrhosis. Br F Dermatol 1987; 116: 265-7.

10 Podesta A, Lopez P, Terg R, et al. Treatment of pruritus of primary biliary cirrhosis with rifampicin. Dig Dis Sci 1991; primary bil $216-20$.

11 Cynamon HA, Andres JM, Iafrate RP. Rifampicin relieves pruritus in children with cholestatic liver disease. Gastroenterology 1990; 98: 1013-6.

12 Acocella G. Pharmacokinetics and metabolism of rifampicin in humans. Rev Infect Dis 1983; 5: S428-32.

13 Miguet JP, Mavier P, Soussy CJ, Dhumeaux D. Induction of hepatic microsomal enzymes after brief administration of rifampicin in man. Gastroenterology 1977; 72: 924-6.

14 Ghent CN, Carruthers SG. Treatment of pruritus in primary biliary cirrhosis with rifampicin. Gastroenterology 1988; 94 : 488-93.

15 Capelle P, Dhumeaux S, Mora M, Feldman G, Berthelot P. Effect of rifampicin on liver function in man. Gut 1972; 13: Effect of

16 Lai S, Singhal SN, Burley DM, Crossley G. Effect of rifampicin and isoniazid on liver function. $B M \mathcal{F} 1972$; $\mathrm{i}$ : 148-50.

17 Grosset J, Leventis S. Adverse effects of rifampicin. Rev Infect Dis 1983; 5: S440-6.

18 Wehrli W. Rifampicin: mechanisms of action and resistance. Ref Infect Dis 1983; 5: S407-10. 